\title{
CONTROLLING THE SIZE OF HEAT AFFECTED ZONE FOR THE AXISYMMETRIC STEEL ELEMENT WITH THE USE OF ANN
}

\begin{abstract}
J. Wróbel ${ }^{*}$, A. Kulawik ${ }^{* *}$
Abstract: The article is a continuation of the considerations on the heat treatment of steel bars. In the paper, a method of automatic velocity choice of a heat source with the use of an artificial neural network is presented. The heat treatment process was performed for the long axisymmetric elements with variable diameters made from medium carbon steel. The two cases of change, linear and step, of the radius of the steel element are considered. It is assumed that the velocity of the heat source is modified in the process and is updated in real time according to the current diameter. The measurement of the diameter is performed at a constant distance from the heat source. The main task of the model is to control the assumed values of temperature at constant parameters of the heat source such as radius and power. The aim of the system, assuming that the heat source has constant values of power and radius, is to provide the established values of temperature on the surface of a quenching element. Therefore, the parameter of the process controlled by the artificial neural network is the velocity of the heat source. The artificial neural network is trained on the basis of results obtained from copyrighted numerical model of thermal phenomena (stationary task). To solve the steady heat transfer equation (axisymmetric task) with a convective term the finite element method is used. To verify the trained artificial neural network numerical simulations are performed based on the solution of the unsteady heat transfer equation.
\end{abstract}

Keywords: heat treatment, moving heat source, artificial neural network, numerical modelling

\section{Introduction}

The application of controllers of the process using an artificial neural network (ANN) is becoming more important in modern industry. The use of the self-reacting systems for variable parameters of the process, such as changing the geometry of the element, has and will be more and more important and will finally lead to a reduction of the human factor. However, such automation requires an initial increase in the costs associated with more measuring devices providing data for intelligent controllers. This is particularly important for low-volume production. The possibility to supervise the process in modern equipment significantly improves the quality of the heat treatment (Canale et al., 2008). The use of work stations including not only the path of the heat source but also having the possibility to determine the distance and angle of inclination to the heating surface is becoming more and more common. Using this type of equipment in the heat treatment for the axisymmetric elements with a variable radius facilitates the production line service and reduces the number of treatments required from the user (Totten, 2006).

The fast control elements in such types of heat treatment equipment may be system-based on artificial neural networks. The important advantage is that neural networks can react correctly on the situation close to the learning patterns (Rojas, 1996, Rutkowski, 2005).

PhD. Eng. Joanna Wróbel: Institute of Computer and Information Sciences, Czestochowa University of Technology, Street Dąbrowskiego 73; 42-201, Częstochowa; PL, joanna.wrobel@icis.pcz.pl

** Assoc. Prof. Adam Kulawik, PhD. Eng.: Institute of Computer and Information Sciences, Czestochowa University of Technology, Street Dąbrowskiego 73; 42-201, Częstochowa; PL,adam.kulawik@icis.pcz.pl 


\section{Solution of the problem}

To determine the learning and testing sets, the application to calculate the temperature fields is developed. These values on the basis of solution of the heat transfer equation with convection term in the axisymmetric coordinates (stationary task) are obtained

$$
\frac{\lambda}{r} \frac{\partial T}{\partial r}+\frac{\partial}{\partial r}\left(\lambda \frac{\partial T}{\partial r}\right)+\frac{\partial}{\partial z}\left(\lambda \frac{\partial T}{\partial z}\right)-\rho C\left(V_{z} \frac{\partial T}{\partial z}\right)=0
$$

where: $\mathrm{T}[\mathrm{K}]$ - temperature, $\lambda=\lambda(\mathrm{T})[\mathrm{W} /(\mathrm{mK})]$ - the thermal conductivity coefficient, $\rho\left[\mathrm{kg} / \mathrm{m}^{3}\right]$ - the density, $\mathrm{C}[\mathrm{J} /(\mathrm{kgK})]$ - the thermal capacity, $\mathrm{V}_{\mathrm{z}}[\mathrm{m} / \mathrm{s}]$ - the velocity of the heat source, $\mathrm{r}$ - is a distance from the symmetry axis.

The numerical simulation of the quenching process was performed for the axisymmetric steel element (Fig. 1). The height of the element was $0.04 \mathrm{~m}$, while the radius of the steel element was selected randomly in the range $0.006-0.014 \mathrm{~m}$. The quenching was modelled by a superficial heat source with a gaussian distribution (Teixeira et al., 2014). It was assumed that the power of the heat source was equal $\mathrm{Q}_{\mathrm{N}}=500 \mathrm{~W}$, radius $\mathrm{R}_{\mathrm{N}}=0.0015 \mathrm{~m}$, whereas the velocity of the heat source was taken from the random value from 0.005 to $0.03 \mathrm{~m} / \mathrm{s}$. On the top surface of the element, the Dirichlet boundary condition $\mathrm{T}=293$ $\mathrm{K}$ was established. It was assumed that heating steel elements rotate to provide uniform distribution of the temperature on the perimeter of the element. In addition, it was assumed that only cases where the maximum temperature on the top surface of the element is between 1000-4000 K will be qualified for the learning and testing sets. The 2000 numerical simulations were performed for various combinations of the velocity of the heat source and the radius of the quenching element.

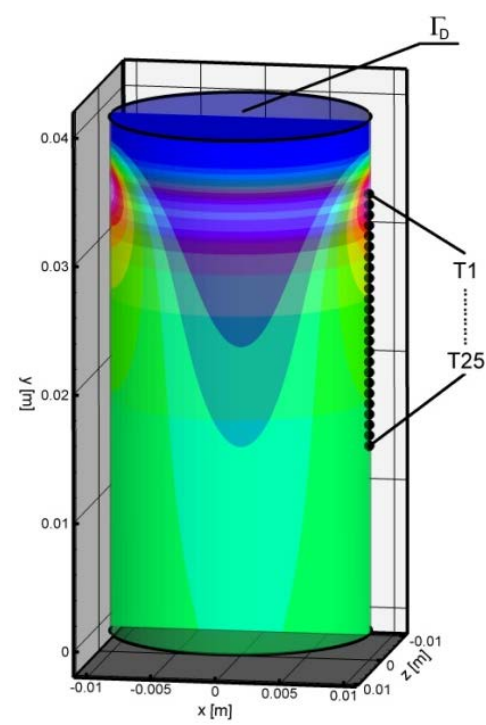

Fig. 1: The geometry of the presented steel element with the sensors. (Wróbel et al., 2015).
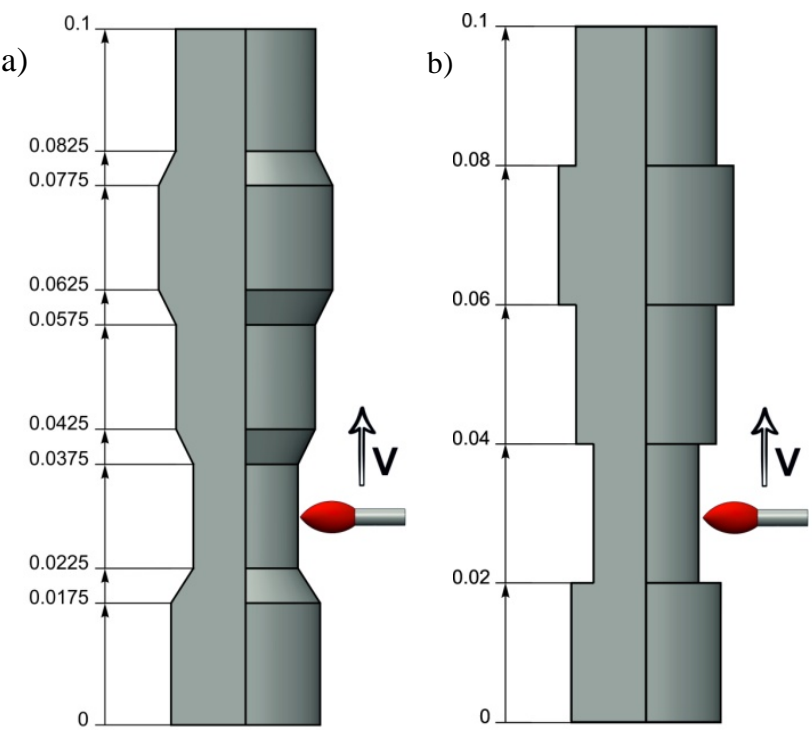

Fig. 2: Considered geometries in the test example: a) geometry No 1, b) geometry No 2.

The data from numerical simulations were divided in half and assigned to the learning and testing sets. From the obtained results, the values of temperature for the 25 sensors located on the boundary of considered element and the radius of the element were selected (Fig. 1). These values were the input data to the ANN. The output parameter of the network was the velocity of the heat source.

The one-way multilayer perceptron with sigmoidal neurons was used. The network was created using one input layer (26 neurons), two hidden layers (20 and 10 neurons) and one output layer (1 neuron) (Wróbel et al., 2015). To train the network, the backpropagation algorithm was used. To increase the speed of the learning neural network, the backpropagation with momentum was applied (Rojas, 1996, Rutkowski, 2005). The momentum coefficient was equal $\alpha=0.7$ and the learning coefficient was $-\eta=0.03$.

To verify the trained artificial neural network, the numerical model of unsteady heat transfer in the axisymmetric coordinates was applied. The numerical tests were performed for the axisymmetric element with a variable diameter. The two cases of change - linear (Fig. 2a) and step (Fig. 2b), of the radius of the steel element were considered. It is assumed that the velocity of the heat source is modified in the process 
and is updated in real time according to the current diameter. The measurement of the diameter is performed at a constant distance from the heat source $(\Delta \mathrm{z}=0)$. The main task of the model is to control the assumed values of temperature (Fig. 3) at constant parameters of the heat source such as radius and power. The initial position of the heat source was situated below the heated element and the start velocity was equal $0.02 \mathrm{~m} / \mathrm{s}$. The initial value of temperature of the steel element the was equal $T_{0}=293 \mathrm{~K}$.

To determine the effectiveness of the used ANN the test was performed. This test consisted in the

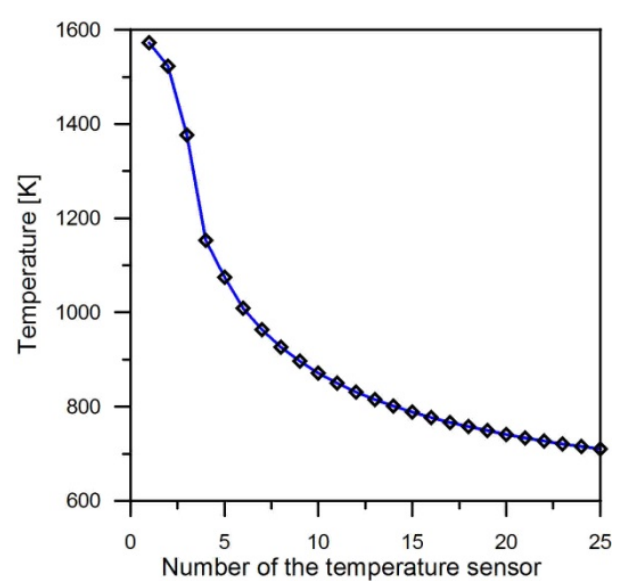

Fig. 3: The input data for the neural network - the required distribution of the temperature (Wróbel et al., 2015).

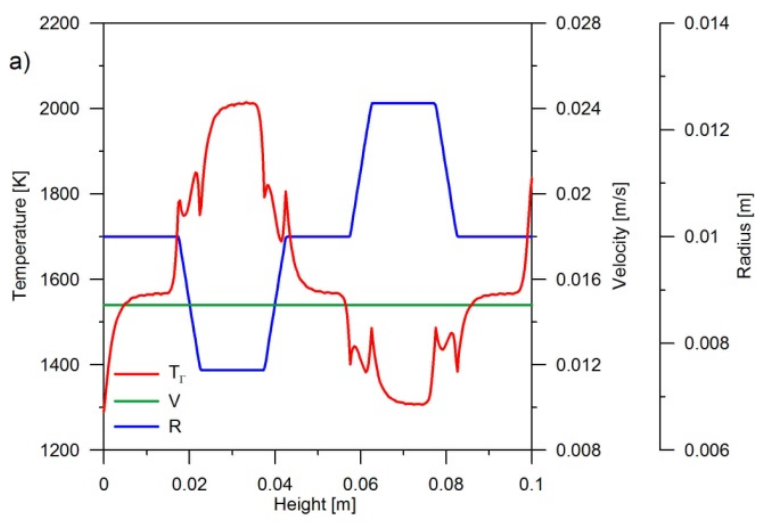

verification the distribution of the value of temperature in the case when an artificial neural network wasn't working and the velocity of the heat source is constant $\mathrm{V}=0.0148 \mathrm{~m} / \mathrm{s}$ (Fig. 4a, 5a). It should be noted that, in both cases, the temperature on the edge increased when the radius of the element decreased, whereas when the radius increased the temperature decreased. The temperature changes had a significantly effect on the width of the heat affected zone (Fig. 6). The size of the heat affected zone (HAZ) was determined by the $\mathrm{A}_{\mathrm{C} 3}$ curve on the level $1058 \mathrm{~K}$. When an artificial neural network was used, it was noticed that on the basis of the temporary value of the radius, the ANN updating the velocity of the heat source, caused temperature changes (Fig. 4b, 5b). The boundary conditions were chosen in such way that the surface layer was not melted. In the place where the radius changed the sudden increased or decreased of temperature was obtained. However, these changes didn't have a significant impact on the depth of the HAZ (Fig. 6).

Fig. 4: Geometry No 1, the changes of temperature for the velocity of the heat source: a) constant, b) determined by ANN.
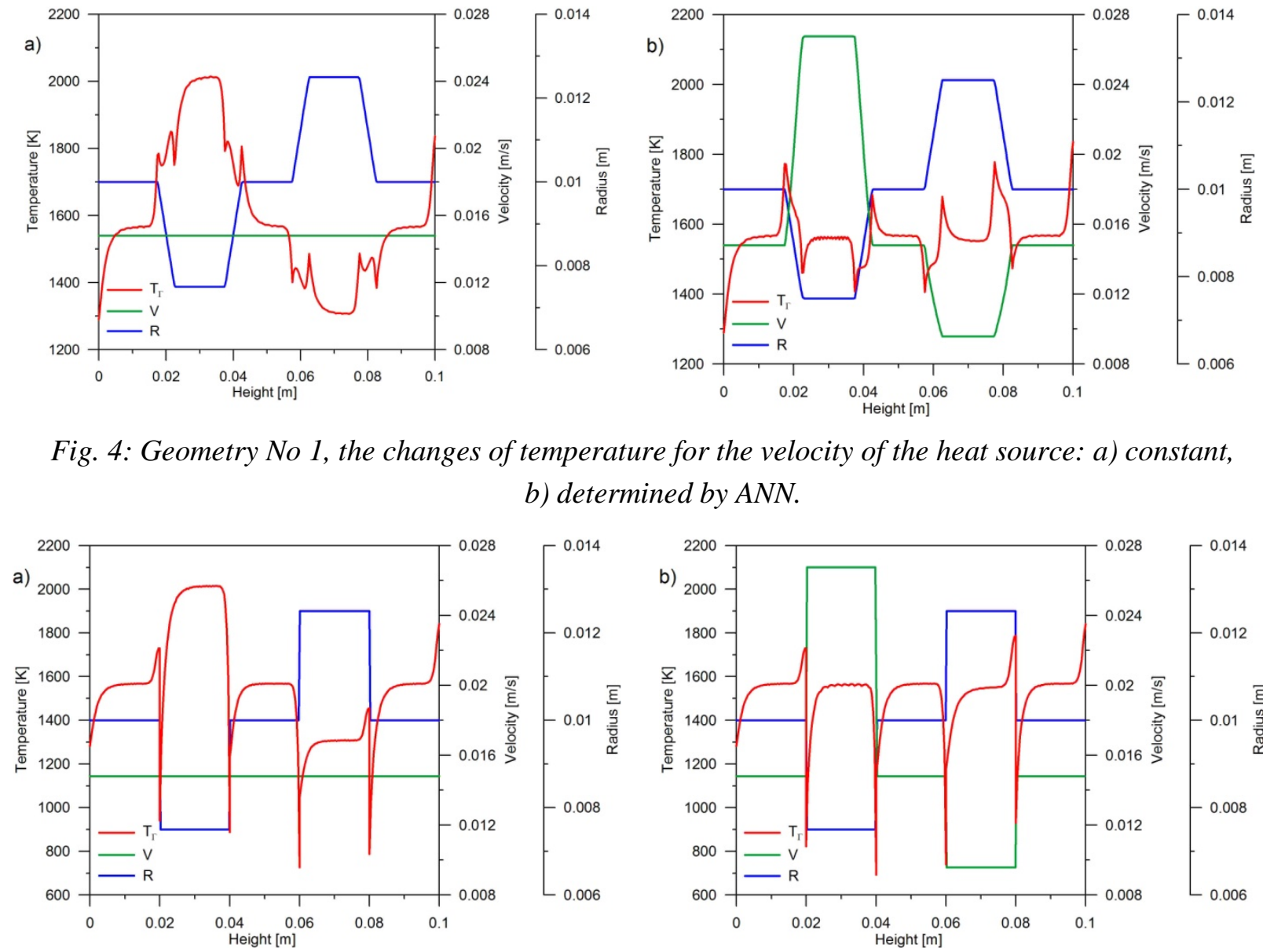

\subsection{4}

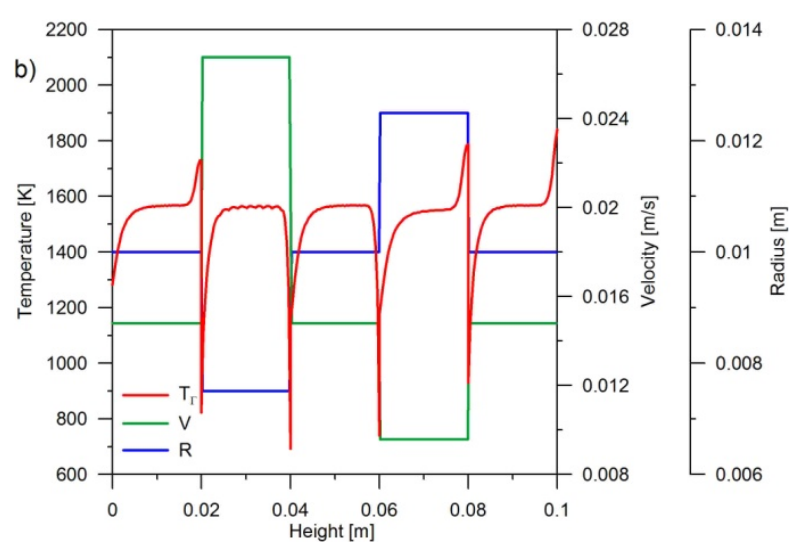

Fig. 5: Geometry No 2, the changes of temperature for the velocity of the heat source: a) constant, b) determined by ANN. 

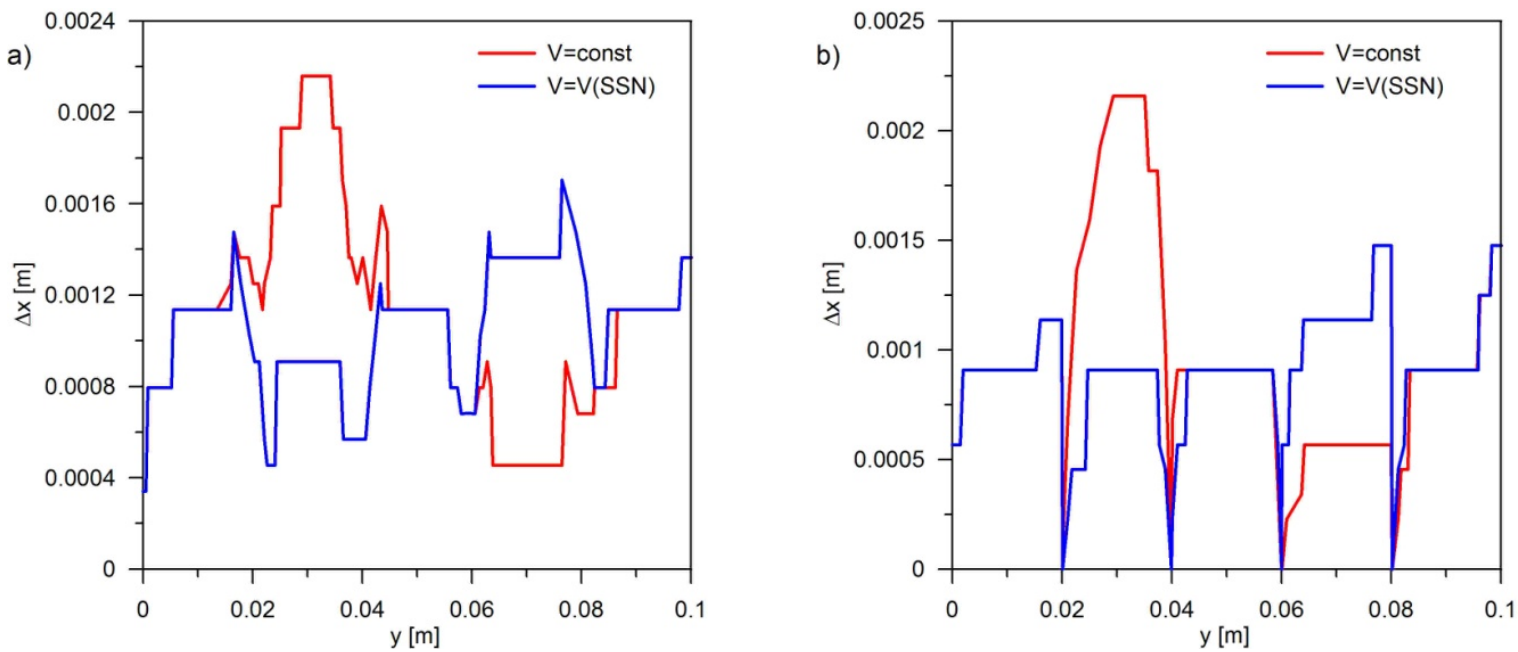

Fig. 6: The size of the heat affected zone: a) geometry No 1, b) geometry No 2.

Figure 6 shows a comparison of the size of the heat affected zone for all analysed velocity of the heat source. In the case of a linear change of radius, the size of HAZ, for the velocity determined by ANN, was $0.00113 \mathrm{~m}$ for radius equalling $0.01 \mathrm{~m}, 0.009 \mathrm{~m}$ for radius $0.0075 \mathrm{~m}$, and $0.0013 \mathrm{~m}$ for radius $0.0125 \mathrm{~m}$ (Fig. 6a). Whereas, for geometry No 2, the size of the HAZ was equal to $0.009 \mathrm{~m}$, only for radius $0.0125 \mathrm{~m}$ was change to $0.0011 \mathrm{~m}$ (Fig. 6b).

\section{Conclusions}

Using the artificial neural network in the control elements of the equipment in the heat treatment allows for effective control of the parameters of this process. In the paper, the control parameter was the velocity of the heat source. The effects of changes of the velocity of the heat source was the maintenance of the established maximum temperature on the surface of the heated element. The artificial neural network correctly reacted on the changes of the radius of the steel element. In the case of changes in the range of the radius of the element or steel grade, artificial neural network calibration must be performed. The presented method of steering of the parameter for the heating process can also be the use of controlling the depth of the heat affected zone. The instability of the maximum temperature on the boundary of areas with a different radius were the result from the assumed position of the radius sensor. The maximum value of the temperature didn't exceed the melting temperature. On the basis of the obtained results, it can be noted that significant problems with keeping the constant width of HAZ occur in the places of radius changes (geometry No 2). Such areas will occur if a maximum temperature limit is set. An appropriate temperature level to ensure the width of the HAZ at the beginning section with the smaller radius caused, as a result overheating of the rest of the sub-area. The step change in the width of HAZ, presented in figure 6 the is due only from the adopted method of visualization and the finite element grid.

\section{Acknowledgement}

The research has been performed within a statutory research BS/PB-1-112-3020/17/P

\section{References}

Canale, L. C. F., Mesquita, R. A., and Totten, G. E. (2008) Failure Analysis of Heat Treated Steel Components. ASM International, United States of America.

Rojas, R. (1996) Neural Network: A systematic introduction. Springer, Berlin.

Rutkowski, L. (2005) Computational Inteligence: methods and techniques. Springer, Berlin.

Teixeria, P., Araújo, D. and Antônio Bragana da Cunha, L. (2014) Study of the gaussian distribution heat source model applied to numerical thermal simulations of TIG welding processes. Science and Engineering Journal, 23, pp. 115-122.

Totten, G. E. (2006) Steel Heat Treatment: Equipment and Process Design. CRC Press, United States of America.

Wróbel, J., Kulawik, A. and Bokota, A. (2015) Artificial neural network to the control of the parameters of the heat treatment process of casting. Archives of Foundry Engineering, 15, 1, pp. 119-124. 